\title{
Years of life lost as a measure of cancer burden on a national level
}

\author{
O T Brustugun *,1,2, B Møller ${ }^{3}$ and $\AA$ Helland ${ }^{1,2}$ \\ ${ }^{1}$ Department of Oncology, Oslo University Hospital-The Norwegian Radium Hospital, Oslo, Norway; ${ }^{2}$ Institute of Clinical \\ Medicine, University of Oslo, Oslo, Norway and ${ }^{3}$ Cancer Registry of Norway, Institute of Population-Based Cancer Research, \\ Oslo, Norway
}

Background: The severity of cancers is often measured in number of deaths. However, number of years of life lost (YLL) may be a more appropriate indicator of impact on society. Here we have calculated the YLL of adult cancers in Norway for the year 2012, as well as for the previous 15-year period.

Methods: Data on age composition, expected remaining years of life, total numbers of deaths and deaths due to cancer were retrieved from the National Census Agency Statistics Norway. YLL were calculated for both sexes aged 25-99 years based on each individual's age at death, and the expected remaining years of life at that age.

Results: Cancer deaths represented $25.8 \%$ of all adult deaths in 2012, with a lower fraction of females $(28.7 \%$ in men and $23.1 \%$ in women), whereas cancer represented $35.2 \%$ of all YLL, with a higher fraction of females (32.8\% in men and $37.8 \%$ in women). Females loose on average more life years to cancer than men (14.9 vs 12.7 years). Average YLL varied from 23.7 (cervical cancer) to 7.9 (prostate cancer). Lung cancer caused almost as many YLL alone (22.1\% of cancer-caused YLL) as colon, prostate and breast cancer combined (23.1\%). From 1997 to 2012, cancer-caused YLL as a fraction of all YLL increased from 32.5\% to 35.2\%, but with major differences among diagnoses.

Conclusions: Cancer is a major and increasing cause of premature deaths, and YLL may be a more accurate measure than number of deaths. Public health efforts and research funding should be explicitly directed at preventing premature deaths.

Cancer deaths are an important public health concern, and are a major cause of premature death worldwide (Burnet et al, 2005; Ferlay et al, 2010). The number of deaths alone does not reflect the complete burden put on the society, as some cancers harm younger people more than others. The number of years of life lost (YLL) therefore depends on the age at death and the number of deaths at each age, and may resolve some of the mismatch of disease impact derived from death numbers alone. YLL data may be more useful in resource allocation and design of prevention programs (Thun et al, 2010; Carter and Nguyen, 2012). Earlier reports have shown that $25-30 \%$ of YLL in western countries are due to cancers (Mariotti et al, 2003; Santric Milicevic et al, 2009; Gènova-Maleras et al, 2011), based on fixed reference ages and subsets of populations. The different cancer subtype contribution to cancer-caused YLL varies substantially, and comprehensive and updated data on a population level are warranted.

Herein we have analysed on a national level the total number of YLL to different cancer diagnoses in the year 2012 as a calculation of number of deaths and the expected remaining life years at each age level. Furthermore, we have compared the cancer-caused YLL with the total amount of premature deaths in our population, as well as the different cancer types to each other. The burden of cancer may change over time both due to cancer-related factors, as incidence and treatment effect changes, and due to changes in other-cause mortality rates (Soneji et al, 2014). Therefore, time trends over the last 15 years are presented. To our knowledge, such a detailed analysis on a national level has not been performed before.

*Correspondence: Dr OT Brustugun; E-mail: otr@ous-hf.no

Received 24 February 2014; revised 26 May 2014; accepted 6 June 2014; published online 1 July 2014 


\section{MATERIALS AND METHODS}

Population demographics. Population-based demographic and death-specific data for the years 1997, 2002, 2007 and 2012 were obtained from the National Institute of Statistics (Statistics Norway).

The total population at the end of 2012 was $5051275 ; 1719477$ males and 1739298 females were 25 years or older.

The life expectancy from birth was 79.4 years for males and 83.4 years for females in 2012, and 75.5 years for males and 81.0 years for females in 1997. Tabulated life expectancies for each age level per sex are updated yearly, and available from Statistics Norway (http://www.ssb.no). For persons 25 years of age, life expectancies were 55.0 years and 58.8 years for men and women, respectively in 2012, whereas at age 80, the corresponding expected remaining years of life were 7.8 years and 9.5 years. In 1997, the corresponding numbers were 51.4 and 56.6 years at age 25 , and 6.6 and 8.5 years at age 80 .

Data collection. Anonymised death records are based on death certificates completed by the physician last in contact with the patient shortly after the time of death and coded according to the 10th revision of the International Classification of Diseases (ICD-10), and collected in the national Cause of Death Register. All diagnoses within chapter $\mathrm{C}$, as well as D32-33 (benign neoplasms of central nervous system (CNS) and meninges) were included (Table 1). Cancer-caused deaths below age 25 and above 99 years are not included in the YLL-analyses due to very few deaths per age level and privacy restrictions. The quality of the Cause of Death Register is regarded high, especially regarding cancer diagnoses, as there is a tight coupling to the Cancer Registry of Norway (CRN, 2014). The system of reporting to the Cancer Registry of Norway was evaluated in 2009, and overall completeness of reporting was estimated at $98.8 \%$ (Larsen et al, 2009). In the

\begin{tabular}{|l|l|}
\hline Table 1. ICD-10 codes for the 18 most frequent cancer types \\
\hline ICD-10 code & Cancer type \\
\hline C00-14 & Head and neck cancer \\
\hline C15 & Oesophageal cancer \\
\hline C16 & Gastric cancer \\
\hline C18-19 & Colon cancer \\
\hline C20-21 & Anorectal cancer \\
\hline C25 & Pancreatic cancer \\
\hline C32-34 & Lung cancer \\
\hline C43 & Malignant melanoma \\
\hline C50 & Breast cancer \\
\hline C53 & Cervical cancer \\
\hline C54 & Uterine cancer \\
\hline C56 & Ovarian cancer \\
\hline C61 & Prostate cancer \\
\hline C64-65 & Kidney cancer \\
\hline C66-68 & Bladder, urinary tract cancer \\
\hline C70-72+ D32-33 & CNS tumours \\
\hline C81-85+C96 & Lymphoma \\
\hline C91-95 & Leukaemias \\
\hline C00-96+D32-34 & All cancers \\
\hline Abbreviations: CNS = central nervous system; ICD = International Classification of Diseases. \\
\hline The 18 cancer types included in the analyses and corresponding ICD-10 codes are shown. \\
\hline & \\
\hline
\end{tabular}

period studied, the proportion of citizens of non-Western origin fluctuated from $2.7 \%$ to $8.1 \%$. In our data set, no subset analysis was conducted based on race, socioeconomic status or geography.

The calculation of YLL was done with the following formula for each type of cancer.

$$
\sum_{i=25}^{99}\left(\# \text { deaths at age } e_{i}\right) \times\left(\text { expected remaining life years at age }{ }_{i}\right)
$$

To provide an estimate of the YLL due to cancer as a fraction of total YLL, we have also calculated a hypothetical total YLL, based on the sum of the products of total number of deaths per age level multiplied with the estimated length of life on each age level. A cause-deleted life table, that is, where the cause that is deleted is the cancer of interest, would imply that persons dying from a specific cause (for example, male lung cancer) had a life expectancy as the general population. As this is not realistic, we have chosen to use the standard life expectancy tables as basis for the calculations (Gellert et al, 2012; Thun et al, 2013).

The average YLL (AYLL) was calculated by dividing the total YLL by the total number of deaths for cancer in total, and for each of the 18 most frequent cancer types for each sex separately.

\section{RESULTS}

Overall deaths. Of a total of 41992 deaths in 2012, cancer caused 10820 (25.8\%), with a lower fraction of females $(28.7 \%$ of all deaths in men and $23.1 \%$ in women). The age distribution is shown (Figure 1). Out of 461 deaths among children and adolescents younger than 25 years, 41 died from cancer $(8.9 \%$ of all deaths in this age group and $0.4 \%$ of all cancer deaths). Sixteen of those were due to CNS tumours, and nine due to leukaemias. Thirteen cancercaused deaths occurred above 99 years of age.

Overall YLL. Based on the total number of deaths at each age level, multiplied by the expected remaining years of life on each level, a hypothetical total of 421047 YLL in age 25-99 years was lost in 2012. In total, 148095 (35.2\%) of these were due to cancer. The fraction of cancer-caused YLL of total YLL was higher in females than in men (37.8\% and $32.8 \%$, respectively). Lung cancer was the main cause (32691 YLL-22.1\% of all cancer-caused YLL) - nearly as many as colon $(15646,10.6 \%)$, breast (10 700, $7.2 \%)$ and prostate cancer $(7979,5.4 \%)$ combined $(34275,23.1 \%)$.

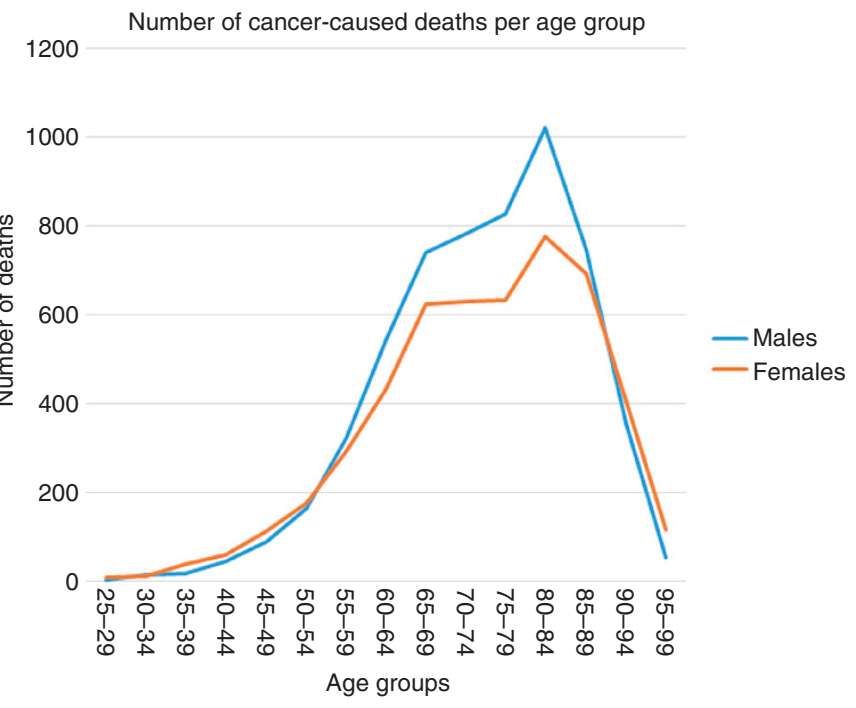

Figure 1. Total number of cancer-caused deaths in Norway in 2012 for both sexes per 5-year age group. 
The YLL for all 18 cancers is shown as percentage of all cancercaused YLL (Figure 2A).

AYLL. AYLL was 13.8 years for cancer in total (148095 YLL/ 10766 deaths), both sexes combined. For the 18 different cancer types, the AYLL varied from 23.7 years (cervical cancer) to 7.9 years (prostate cancer) (Figure 2B). Deaths among female-specific cancers as cervical, breast, ovarial and uterine cancers occured in lower ages than the male-specific or -predominant cancers as prostate, bladder and oesophageal cancers, contributing to a higher AYLL in females than in males (14.9 and 12.7 years, respectively).

YLL of cancer subtypes. The YLL of each cancer can be expressed as a percentage of the total YLL to cancers, and related to the number of deaths (mortality) of each cancer as a percentage of the total number of deaths of cancer. The results of these calculations indicate the relative difference, that is, the degree of imbalance between the YLL parameters and mortality parameters, and are shown in Figure 3A. For most cancer types, the YLL (\%)/mortality (\%) ratio is close to 1 , but ranged from 0.58 (prostate, low relative YLL) to 1.72 (cervical cancer, high relative YLL) (Figure 3B). Lung cancer, responsible for the by far highest YLL percentage and deaths percentage, had a ratio of 1.07 , which indicate that both YLL and mortality are high.
For the four major cancer diagnoses, distinct differences in age distribution of YLL are seen when plotting YLL against age (Figure 4). Colon cancer claims YLL predominantly in high ages, especially among women, and this is even more evident for prostate cancer which reaches peak YLL in the 80-84-year age group. In women up to the age of 55 , breast cancer is representative of the largest loss of life years, but thereafter, lung cancer is the major cancer cause of YLL loss also in women. For lung cancer, the highest YLL is found around 70 years for both sexes, whereafter a steep decrease is seen, in line with a decline in mortality rates in the highest age groups for lung cancer (data not shown).

Changes over time. Over the last 15 years, the amount of deaths due to cancers in age 25-99 years as a fraction of all deaths in this group increased from $24.3 \%$ to $26.2 \%$, whereas cancer-caused YLL as a fraction of total YLL increased from $32.5 \%$ to $35.2 \%$ (Figure 5A). The relative contribution of the main cancers has changed over the period (Figure 5B). Among females, both the number of deaths caused by lung cancer as well as the YLL have increased substantially as a fraction of total cancer, whereas both parameters have decreased for breast cancer (Figure 5B). Also among men, a similar trend is evident-increase, althoug moderate, in lung cancer parameters and decrease in prostate cancer (Figure 5B). The gap between the death curve and the YLL

A 25 YLL per cancer type as fraction of cancer-caused YLL

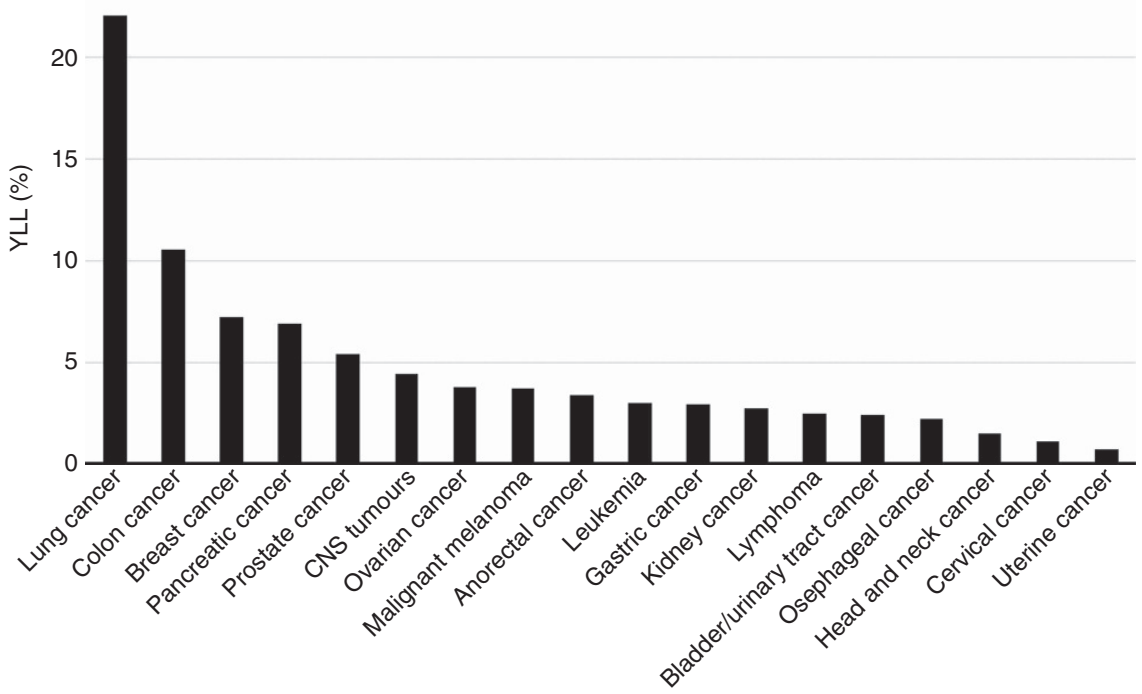

B

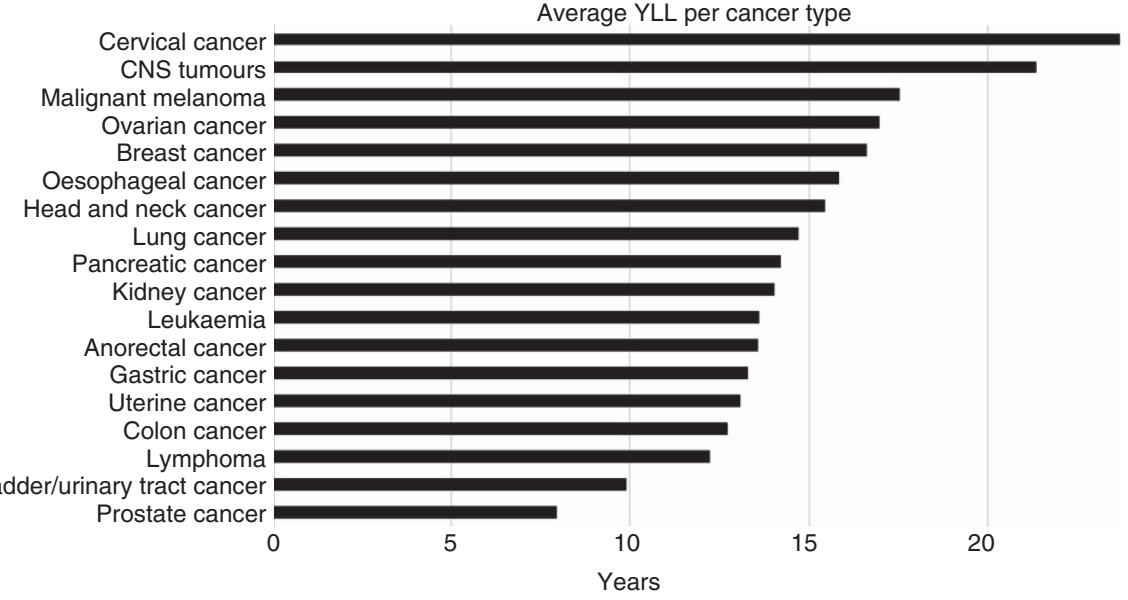

Figure 2. Percentage YLL of total cancer-caused YLL in Norway in 2012 for 18 most frequent cancer types. Percentage YLL of total cancer-caused YLL in Norway 2012 for 18 most frequent cancer types, both sexes combined (A). Average YLL of the 18 most frequent cancer types (B). 
A
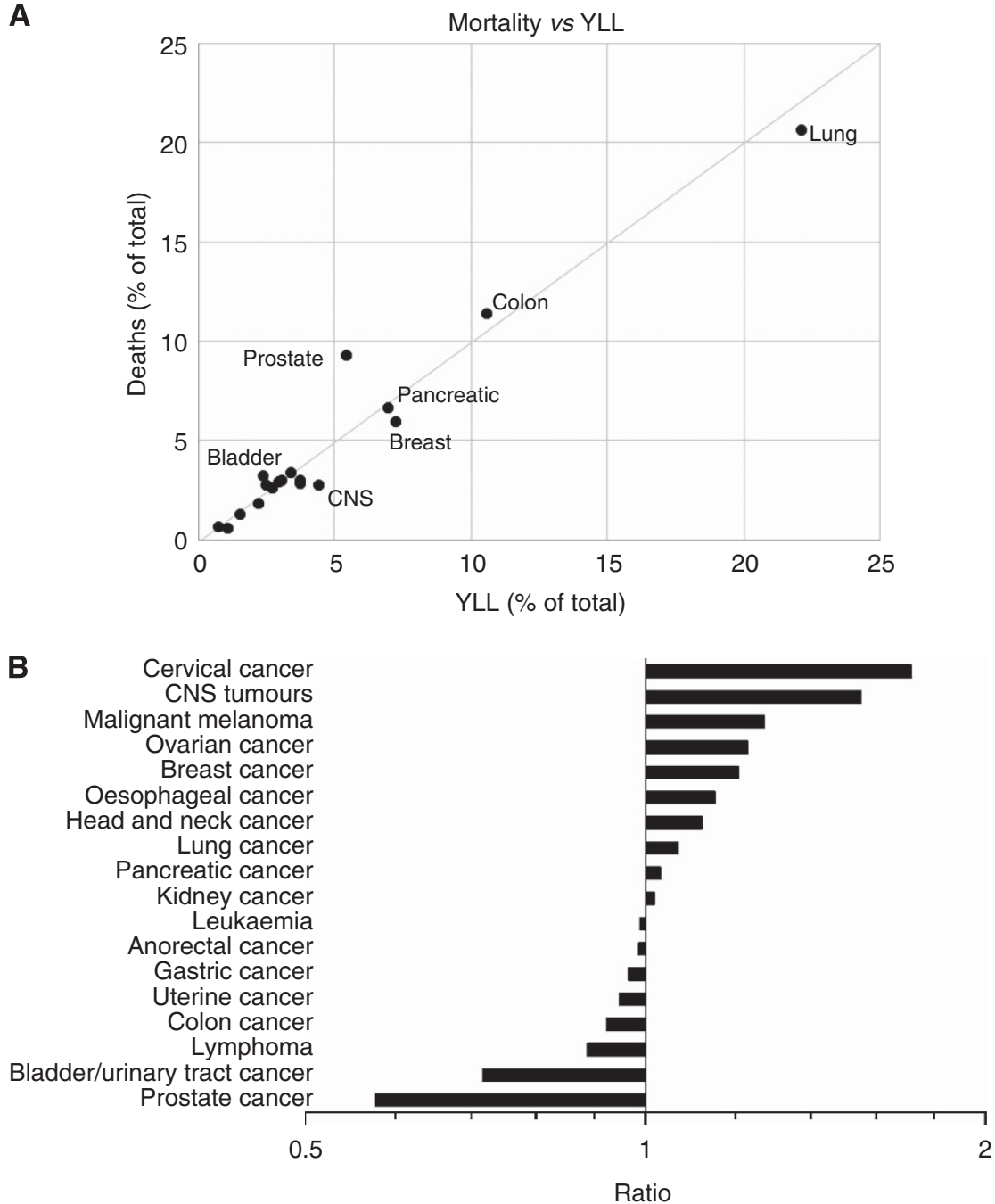

Figure 3. Number of deaths of individual cancer types in Norway in 2012 as a percentage of all cancer deaths ( $y$ axis) and total YLL of the same cancer type as a percentage of all cancer-related YLL (x axis). The ratio of YLL (\%) to deaths (\%) (A) and the ratios (log scale) of YLL (\%)/mortality (\%) are shown for the 18 most frequent cancers (B).
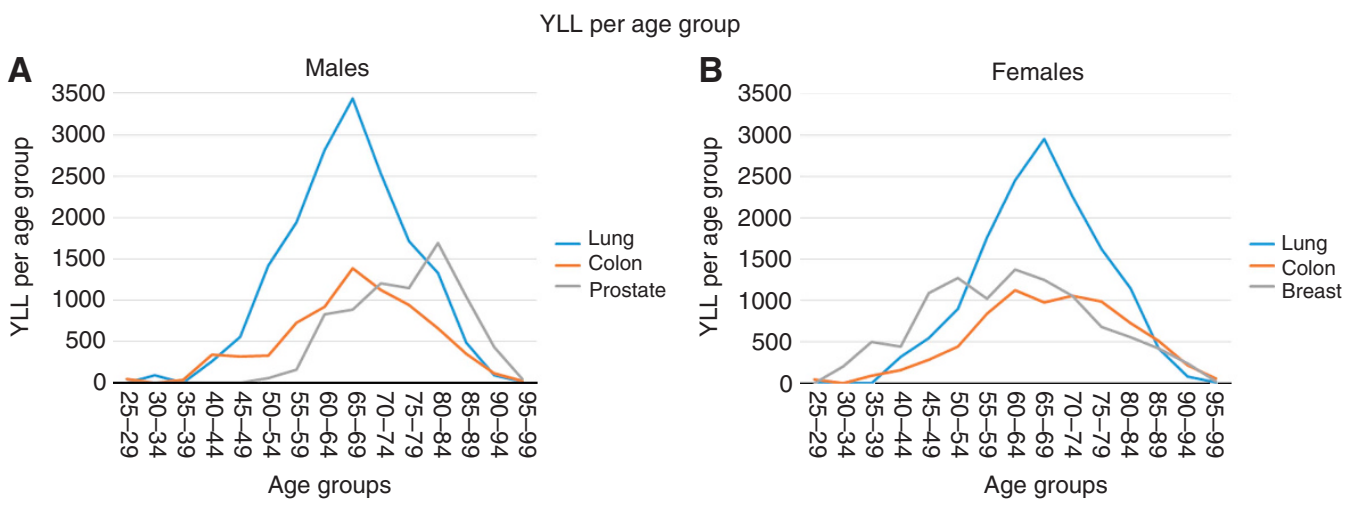

Figure 4. YLL caused by lung, colon and prostate cancer for men (A), and by lung, breast and colon cancer for women (B), shown for 5-year age groups from 25 to 99 years in Norway in 2012.

curve decreases with time for both female lung cancer and breast cancer, implying that death caused by these diagnoses now occurs at higher age than before. The relative increase or decrease of YLL caused by the individual cancers, as well as cancer in total, from 1997 to 2012 is shown for both sexes, shown as the ratio of $\mathrm{YLL}_{2012}: \mathrm{YLL}_{1997}$ (Figure 6). Cancer-caused death in males below
25 as a fraction of all cancer-caused death has declined from $0.67 \%$ ( 38 of 5713 ) to $0.30 \%$ (17 of 5762) in the 15 -year period, wherease for females, no clear change is seen (27 deaths below 25 out of 5713 in total in $1997(0.54 \%)$, vs 24 out of $5058(0.47 \%)$ in 2012. The total number of deaths below 25 has declined substantially, from 454 deaths among male and 254 among female in 1997 to 293 and 
A $40 \quad$ Cancer-caused vs total deaths and YLL
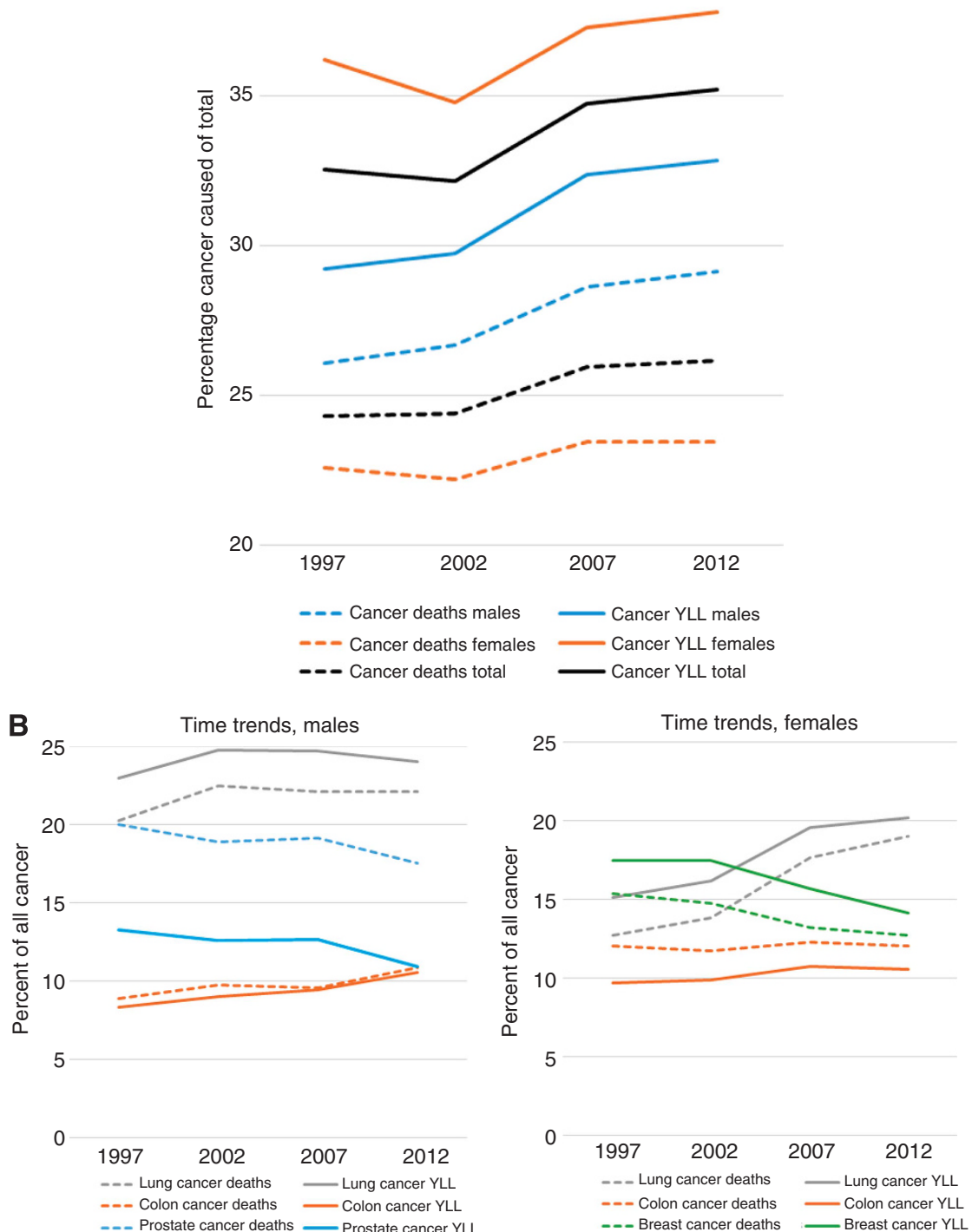

Figure 5. Cancer-caused deaths. Cancer-caused deaths as percentage of all deaths for each sex, and combined (dotted lines) and cancer-caused YLL as percentage of all YLL for each sex, and combined (solid lines) in Norway for the period 1997-2012 (A). Deaths and YLL caused by lung, colon or prostate cancer for men (left panel), and by lung, colon and breast cancer for women (right panel) as percentage of all cancer deaths and YLL per sex in Norway in the period 1997-2012 (B).

168 , respectively in 2012 , thus the fraction of cancer-caused death in females aged 0-24 years has increased, whereas it has been stable in males.

\section{DISCUSSION}

We have, on a national level, shown the relative contribution to premature deaths due to a number of cancers, as well as to cancer in total, expressed as YLL. A third of all YLL are due to cancer, and the YLL due to lung cancer equals those due to breast, colon and prostate cancer combined. A cancer death represented on average 13.0 YLL, but large variations among cancers are evident.

Interestingly, we have shown that even though the number of cancer-caused deaths were higher among men than among females, females lost substantially more YLL than men. This cannot only be explained with a longer life expectancy among females than among males, as below the age of 55, more women than men died from cancer.

Even though cancer risk is inheritably linked to higher age, a number of cancers harm relatively young people. Focus should therefore not only be on the deadly cancers in terms of absolute death toll, but also taking into account the numbers of life years lost due to the disease (Liu et al, 2013). Research spending has been shown to be more discrepant if cancer burden measured as YLL, and not only death numbers, forms the basis of comparison of spending and severity (Carter and Nguyen, 2012). Cancer deaths also have a substantial impact on general life expectancy in the population. One of four (25.8\%) dies of cancer, with an average loss of life years of 13.0 years. This means that cancer diseases reduce general life expectancy with over 3 years in the Norwegian population. However, as the cancer-caused deaths are included in the current life tables, the impact on life expactancy would be higher than the abovementioned indication, but cannot be calculated exactly. This is due to the fact that it is unrealistic that 

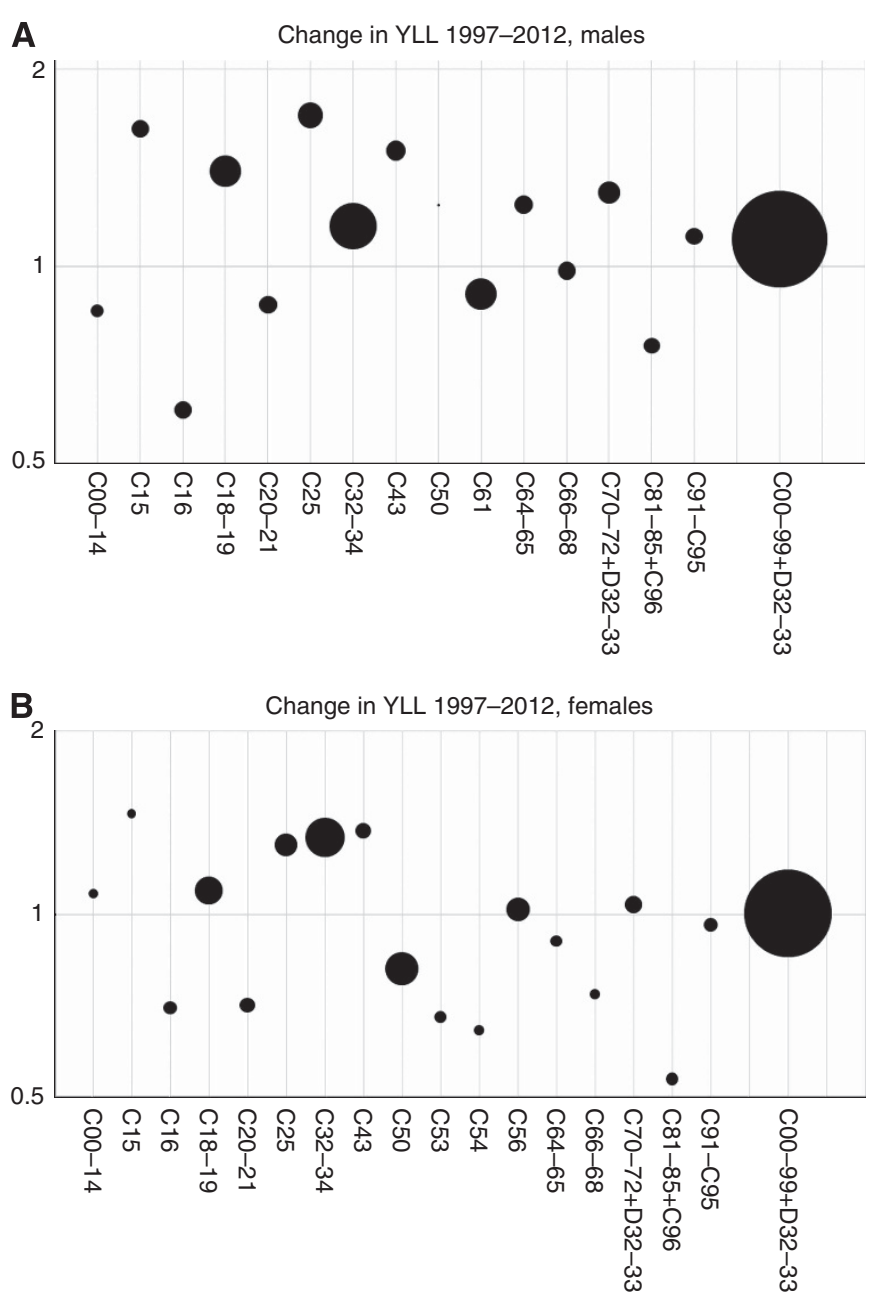

Figure 6. The relative increase or decrease in YLL from 1997 to 2012 for the individual diagnoses as well as cancer in total. The relative increase or decrease in YLL from 1997 to 2012 for the individual diagnoses as well as cancer in total for males $(\mathbf{A})$ and females (B). The size of the balls reflects the magnitude of YLL in 2012 caused by the respective diagnoses.

cancer patients would have had a survival similar to non-cancer patients if the cancer cause had been left out.

Lung cancer is one of the most frequent cancers worldwide in both sexes. Smoking habits in many western countries have changed during the past decades, but it still retains the most important health hazard (Schroeder, 2013). Prevention via smoking restrictions may have significant impact on longevity (Jha et al, 2013; Liu et al, 2013). Hypothesising that half of lung cancer deaths (which on average caused 14 YLL and represented $7.4 \%$ of all YLL) were eradicated, general life expectancy would increase with around half a year (14 AYLL $\times 7.4 \% / 2)$. Life expectancy from birth in Norway in 2012 was 0.4 years lower than in Sweden (81.8 vs 81.4) (Statistics Sweden, http:// www.scb.se). Lung cancer incidence in Sweden is approximately half compared with Norway (Engholm et al, 2010), and this difference alone may therefore explain the lower life expectancy in Norway.

Prostate cancer, the most prevalent cancer diagnosis among men, is responsible for $18.1 \%$ of all cancer deaths in males, but only $11.7 \%$ of all cancer-related YLL, due to the low AYLL of 7.9 yearsthe lowest of the major cancers, in line with what has been reported earlier (Burnet et al, 2005). However, the time from diagnosis to death of any cause is long in prostate cancer, which makes disability-adjusted life years, which are the sum of YLL and years lived with disability relevant (Murray, 1994; Soerjomataram et al, 2012).

On the other hand, deaths caused by cervical cancer as well as CNS tumours, occur in relative young ages. Although the numerical number of deaths is moderate, the low age contributes to a relative high YLL. Interestingly, CNS tumours were also the most frequent cancer-caused death among persons younger than 25 years, which in this study are not included in the YLL calculations. This means that the total YLL caused by CNS tumours is higher, albeit marginal due to the low frequency, than reported herein.

Other studies that have reported cancer-caused YLL have found comparable, but generally somewhat lower AYLL estimates (NIH, 2001; Burnet et al, 2005; Pham et al, 2009; Santric Milicevic et al, 2009). This may partly be due to lower general life expectancies in the populations under study, and the use of standardized life expectancy which impedes direct comparison with our study. To our knowledge, the present study is the first on a complete, detailed nationwide data set, where every adult death up to the age 99 years in the year 2012 were put into the calculations together with up-todate life expectancy tables. Furthermore, detailed cause-of-death information is also provided for elderly people in our population. This may in sum provide a more correct estimate of cancer-caused YLL.

There has been a substantial increase in cancer incidence in Norway over the last 15 years (from approximately 250:100 000 for females and 300:100000 for males to now approximately 300:100 000 for females and 370:100000 for males (CRN, 2014). Furthermore, mortality due to non-cancer causes as cardiovascular diseases has decreased markedly in Western countries over this period (Berg et al, 2014). Still the increase in cancer-caused YLL as a fraction of total YLL has been only moderate. This is probably mainly due to progress in treatment, as well as the fact that most of the incidence comes in high ages, where impact on YLL is limited (Soneji et al, 2014). Contrasting these findings is the worrisome increase in lung cancer-caused YLL among females, reflecting the considerable increase in smoking behaviour among women of all ages that has only recently started to abate (Statistics Norway, http://www.ssb.no). A reduction in cancer mortality in the age group below 25 is seen over the last 15 years, in line with what is seen in most Western countries (Kowalczyk et al, 2014). However, due to a decrease in all-caused death in this group, a relative increase in cancer-related mortality is in fact seen in children and adolescents up to 25 years, especially among females.

A limitation of the study is the single-nation aspect and the use of non-standardized life expectancy assumptions. But the general life expectancy, as well as the cancer incidence panorama in Norway is comparable with most other Western countries, thus making the information in this study generalisable to a large extent. Disability-adjusted life years as well as quality-adjusted life years are not calculated in the present report, as this implies a detailed knowledge of the period of disease before death, which in our opinion will be of limited certainty in a population-based study. However, disability-adjusted life years and quality-adjusted life years applied to a well-defined cohort of cancer patients, where detailed and objective quality of life measures are collected, may provide information that complement the population-based metrics as YLL.

There are a number of parameters that together depict the severity of disease, and that in sum may provide guidance for costeffective preventive strategies, research efforts and predict drivers of health care costs. We believe YLL is an important measure that should be taken into account, and we have herein shown that this parameter may skew the focus towards female-dominant cancers as well as smoking-related cancer.

In conclusion, we have shown that cancer is a major cause of premature deaths in Norway, and that reporting YLL accentuates 
the effect of cancer put on the society. Public health efforts and research funding allocation should be more explicitly directed at preventing premature death. Addressing prevention of the major causes of YLL-especially lung cancer-may thus result in substantial impact on general life expectancy.

\section{ACKNOWLEDGEMENTS}

Help in data retrieval from Statistics Norway by Øystein KramerJohansen is highly appreciated. This work was supported by the South-Eastern Norway Regional Health Authority to OTB and ÅH.

\section{CONFLICT OF INTEREST}

The authors declare no conflict of interest.

\section{REFERENCES}

Berg J, Björck L, Lappas G, O’Flaherty M, Capewell S, Rosengren A (2014) Continuing decrease in coronary heart disease mortality in Sweden. BMC Cardiovasc Disord 14: 9.

Burnet NG, Jefferies SJ, Benson RJ, Hunt DP, Treasure FP (2005) Years of life lost (YLL) from cancer is an important measure of population burden-and should be considered when allocating research funds. Br J Cancer 92(2): 241-245.

Carter AJ, Nguyen CN (2012) A comparison of cancer burden and research spending reveals discrepancies in the distribution of research funding. BMC Public Health 12: 526.

CRN (2014) Cancer in Norway 2012. Cancer Registry of Norway: Oslo.

Engholm G, Ferlay J, Christensen N, Bray F, Gjerstorff ML, Klint A, Køtlum JE, Olafsdóttir E, Pukkala E, Storm HH (2010) NORDCAN-a Nordic tool for cancer information, planning, quality control and research. Acta Oncol 49(5): 725-736.

Ferlay J, Shin HR, Bray F, Forman D, Mathers C, Parkin DM (2010) Estimates of worldwide burden of cancer in 2008: GLOBOCAN 2008. Int J Cancer 127(12): 2893-2917.

Gellert C, Schöttker B, Brenner H (2012) Smoking and all-cause mortality in older people: systematic review and meta-analysis. Arch Intern Med 172(11): 837-844.

Gènova-Maleras R, Catalá-López F, de Larrea-Baz NF, Álvarez-Martín E, Morant-Ginestar C (2011) The burden of premature mortality in Spain using standard expected years of life lost: a population-based study. BMC Public Health 11: 787.

Jha P, Ramasundarahettige C, Landsman V, Rostron B, Thun M, Anderson RN, McAfee T, Peto R (2013) 21st-century hazards of smoking and benefits of cessation in the United States. N Engl J Med 368(4): 341-350.

Kowalczyk JR, Samardakiewicz M, Fitzgerald E, Essiaf S, Ladenstein R, Vassal G, Kienesberger A, Pritchard-Jones K (2014) Towards reducing inequalities: European Standards of Care for Children with Cancer. Eur J Cancer 50(3): 481-485.

Larsen IK, Smastuen M, Johannesen TB, Langmark F, Parkin DM, Bray F, Moller B (2009) Data quality at the Cancer Registry of Norway: an overview of comparability, completeness, validity and timeliness. Eur J Cancer 45(7): 1218-1231.

Liu PH, Wang JD, Keating NL (2013) Expected years of life lost for six potentially preventable cancers in the United States. Prev Med 56(5): 309-313.

Mariotti S, D’Errigo P, Mastroeni S, Freeman K (2003) Years of life lost due to premature mortality in Italy. Eur J Epidemiol 18(6): 513-521.

Murray CJ (1994) Quantifying the burden of disease: the technical basis for disability-adjusted life years. Bull World Health Organ 72(3): 429-445.

NIH (2001) Stat bite: average years of life lost from cancer. J Natl Cancer Inst 93(5): 341.

Pham TM, Fujino Y, Ide R, Tokui N, Kubo T, Mizoue T, Ogimoto I, Matsuda S, Yoshimura T (2009) Years of life lost due to cancer in a cohort study in Japan. Eur J Public Health 19(2): 189-192.

Santric Milicevic M, Bjegovic V, Terzic Z, Vukovic D, Kocev N, Marinkovic J, Vasic V (2009) Serbia within the European context: an analysis of premature mortality. Popul Health Metr 7: 12.

Schroeder SA (2013) New evidence that cigarette smoking remains the most important health hazard. N Engl J Med 368(4): 389-390.

Soerjomataram I, Lortet-Tieulent J, Parkin DM, Ferlay J, Mathers C, Forman D, Bray F (2012) Global burden of cancer in 2008: a systematic analysis of disability-adjusted life-years in 12 world regions. Lancet 380(9856): 1840-1850.

Soneji S, Beltrán-Sánchez H, Sox HC (2014) Assessing progress in reducing the burden of cancer mortality, 1985-2005. J Clin Oncol 32(5): 444-448.

Thun MJ, Carter BD, Feskanich D, Freedman ND, Prentice R, Lopez AD, Hartge P, Gapstur SM (2013) 50-year trends in smoking-related mortality in the United States. N Engl J Med 368(4): 351-364.

Thun MJ, DeLancey JO, Center MM, Jemal A, Ward EM (2010) The global burden of cancer: priorities for prevention. Carcinogenesis 31(1): 100-110.

(c) (1)(2) This work is licensed under the Creative Commons (c) Attribution-NonCommercial-Share Alike 3.0 Unported License. To view a copy of this license, visit http://creativecommons. org/licenses/by-nc-sa/3.0/ 